\title{
Etalonnage de cristaux organiques auprès du rayonnement synchrotron de Super ACO
}

\author{
L. Beck, C. Bizeuil et P. Stemmer \\ CEA, Service CEM, BP. 12, 91680 Bruyères-le-Châtel, France
}

\begin{abstract}
The spectrometric properties of several hydrophthalate crystals (KAP, RbAP, TlAP and CsAP) are studied by using the synchrotron radiation. We especially investigate cesium hydrophthalate for which little information is available in the literature.

We present experimental measurements of rocking curves at $1000 \mathrm{eV}$ and $2500 \mathrm{eV}$ performed on the SB3 beamline of Super ACO storage ring (LURE-Orsay).
\end{abstract}

\section{INTRODUCTION}

La ligne SB3 de Super ACO a été développée pour l'étalonnage d'optiques et de détecteurs pour le rayonnement $X$. Dans le cadre des optiques, nous nous sommes particulièrement intéressés à la caractérisation des cristaux de fine bande passante, qui grâce à leur pouvoir résolutif important sont utilisés en spectrométrie [1,2].

Nous présentons l'étalonnage de cristaux d'acides phtaliques (TlAP, RbAP, KAP et CsAP) à 1000 et $2500 \mathrm{eV}$.

\section{INSTRUMENTATION ET SELECTION SPECTRALE}

La ligne de lumière est composée principalement d'un monochromateur et d'un goniomètre $\Theta / 2 \Theta$. Le monochromateur comprend deux cristaux parallèles permettant de sélectionner des bandes d'énergie comprises entre $0.5 \mathrm{à} 8 \mathrm{keV}$. La direction du faisceau de sortie est maintenue horizontale et fixe grâce à une translation du cristal inférieur. Nous avons utilisé des cristaux de béryls $(2 \mathrm{~d}=15.95 \AA)$ à $1000 \mathrm{eV}$ et des cristaux de silicium $(2 \mathrm{~d}=6.27 \AA)$ à $2500 \mathrm{eV}$. Leur résolution aux énergies de travail mentionnées cidessus sont respectivement de $0.28 \mathrm{eV}$ et $0.195 \mathrm{eV}$. Le goniomètre est composé de deux platines goniométriques permettant d'orienter l'optique à analyser d'un angle $\Theta$ et le détecteur d'un angle $2 \Theta$ par rapport au faisceau incident.

Deux translations de l'échantillon permettent de balayer la surface étudiée. Les précisions sur les rotations sont de $5.10^{-5}$ degré et sur les translations de $1 \mu \mathrm{m}$. Le détecteur est un compteur proportionnel à flux gazeux (mélange Ar 90/méthane 10) travaillant à 500 mbar. 


\section{RESUltats}

Par rotation de chaque cristal autour de l'angle de Bragg des courbes de réflexion (ou rocking curves) on été mesurées en polarisation $\sigma$. Elles sont représentées sur la figure 1 pour les 4 cristaux testés à $2500 \mathrm{eV}$. Nous avons observé les résultats suivants aux deux énergies étudiées. Le cristal de KAP est le cristal qui présente la meilleure résolution spectrale tandis que CsAP a la plus large bande passante. Compte tenu de sa bonne réflectivité-pic ce cristal semble donc parfaitement adapté à des expériences où un fort pouvoir réflecteur est demandé. En revanche, le cristal de KAP est le meilleur cristal pour la spectrométrie de haute résolution.

Ces résultats expérimentaux ont été comparés à des simulations générées par le logiciel de tracé de rayons Shadow [3] dont les développements sur les cristaux organiques sont présentées à cette conférence [4]. L'accord est excellent à $2500 \mathrm{eV}$. En revanche, à $1000 \mathrm{eV}$ on observe l'élargissement de la rocking curve dû au fait que les cristaux de béryls ne sont pas assez résolutifs. Nous avons donc dû ensuite recourir à la déconvolution des courbes pour déduire la bande passante des cristaux organiques [5]. Ces expériences combinées aux simulations ont permis de déterminer les propriétés du cristal de CsAP pour lequel aucune donnée spectrométrique n'avait encore été publiée. Elles ont aussi permis de calculer la distance entre les plans réticulaires $(2 \mathrm{~d}=25.56 \AA)$ et ainsi de confirmer la structure proposée par Teplova et al. [6].

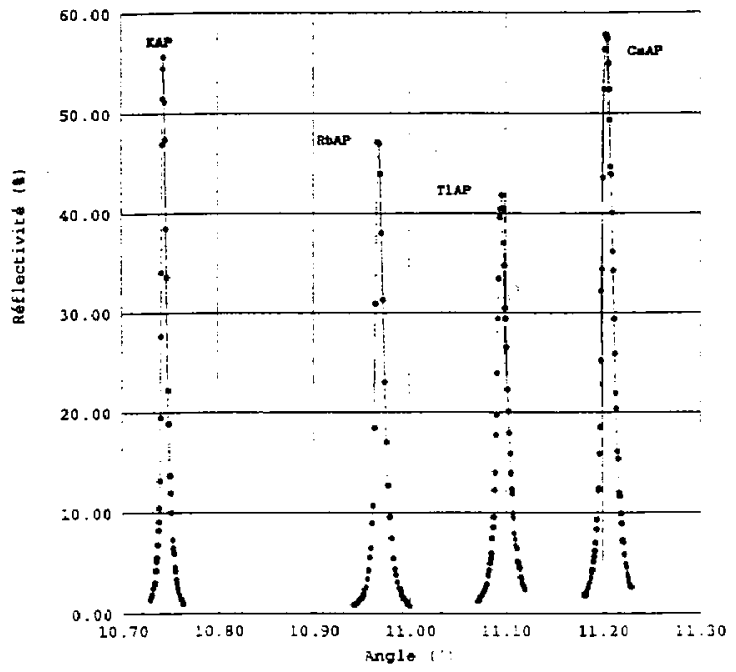

Figure 1 : Courbes de réflexion des cristaux d'acides phțaliques à $2500 \mathrm{eV}$.

\section{Remerciements}

Les auteurs remercient M. Marmoret du CEA et M. Robelin de la Société Crismatec pour l'obtention des cristaux.

\section{Rêférences}

[1] P.G. Burkhalter et al., J. Appl. Phys. 52(7) (1981)

[2] A. J. Burek et al., Astrophys. J. 191, 533 (1974)

[3] B. Lai et F. Cerrina, NIM A246, 337, (1986)

[4] F. Legrand et al., voir cette revue

[5] L. Beck, P. Stemmler and F. Legrand, to be published in the proceedings of the SRI Conference, Stony Brook 18-22 July 1994, Review of Scientific Instrumrnts (1994)

[6] T. B. Teplova, Sov. Phys. Crystallogr. 35(1) (1990) 